\title{
Optomechanically induced transparency, amplification, and Fano resonance in a multimode optomechanical system with quadratic coupling
}

Yongchao Zhang ${ }^{1}$, Zhipeng Zhu' ${ }^{1}$, Yuanshun Cui ${ }^{1}$, Hualing Yu ${ }^{1}$, Cheng Jiang ${ }^{1 *}$ and Xiufeng Cao ${ }^{2^{*}}$

\author{
"Correspondence: \\ chengjiang8402@163.com; \\ xfcao@xmu.edu.cn \\ 'Physics Department, Jiangsu Key \\ Laboratory of Modern \\ Measurement Technology and \\ Intelligent Systems, Huaiyin Normal \\ University, Huai'an, 223300, China \\ 2Department of Physics, Xiamen \\ University, Xiamen, 361005, China
}

\begin{abstract}
We explore the optical response of a multimode optomechanical system with quadratic coupling to a weak probe field, where the cavity is driven by a strong control field and the two movable membranes are, respectively, excited by weak coherent mechanical driving fields. We study the two cases that the two movable membranes are degenerate and nondegenerate. For the degenerate case, it is shown that only one transparency window occurs and the transition between optomechanically induced transparency and Fano resonance can be realized by tuning the cavity-control field detuning. For the nondegenerate case, two transparency windows are observed and the absorption spectrum can switch between a single Fano resonance and double Fano resonances. Furthermore, we show that the output probe field can be greatly amplified or completely suppressed due to the complex interference effect by tuning the amplitude and phase of the mechanical driving fields. Our results can be extended to the optomechanical system with multiple membranes, which enables us to control the light propagation more flexibly.
\end{abstract}

Keywords: Optomechanically induced transparency; Amplification; Fano resonance; Multimode optomechanical system; Quadratic coupling

\section{Introduction}

Optomechanical system usually consists of the electromagnetic cavity and the mechanical oscillator coupled by radiation pressure [1-3]. In the past decades, both linear and quadratic optomechanical coupling have been under extensive exploration, where the cavity resonance frequency linearly and quadratically depends on the displacement of the mechanical oscillator, respectively. In particular, tremendous progresses have been made in optomechanical systems, such as ground state cooling of the mechanical oscillator $[4,5]$, optomechanical squeezing of light and mechanical motion [6-9], quantum entanglement between mechanical oscillators $[10,11]$. Furthermore, the optical response of the optomechanical system can exhibit some interesting phenomena by driving the cavity under

(c) The Author(s) 2021. This article is licensed under a Creative Commons Attribution 4.0 International License, which permits use, sharing, adaptation, distribution and reproduction in any medium or format, as long as you give appropriate credit to the original author(s) and the source, provide a link to the Creative Commons licence, and indicate if changes were made. The images or other third party material in this article are included in the article's Creative Commons licence, unless indicated otherwise in a credit line to the material. If material is not included in the article's Creative Commons licence and your intended use is not permitted by statutory regulation or exceeds the permitted use, you will need to obtain permission directly from the copyright holder. To view a copy of this licence, visit http://creativecommons.org/licenses/by/4.0/. 
different conditions, including optomechanically induced transparency (OMIT) [12-19], optomechanically induced amplification (OMIA) [20, 21], and Fano resonance [22, 23]. OMIT is the analog of electromagnetically induced transparency (EIT) [24, 25], which results from the destructive interference between different excitation pathways in atomic vapors and various solid-state systems [26-29]. Recently, different types of OMIT, such as nonlinear OMIT [30-32], two-color OMIT [33], vector OMIT [34], reversed OMIT [35-37], and nonreciprocal OMIT [38, 39], have been extensively explored. Besides the nonreciprocal OMIT [39], the spinning optomechanical system also provides a platform to study quantum effects such as nonreciprocal photon blockade [40] and nonreciprocal optomechanical entanglement [41]. Closely related to EIT, the Fano resonance with asymmetric line shape was first explained by Ugo Fano in terms of the interference of a narrow discrete resonance with a broad spectral line or continuum [42, 43]. These phenomena can have potential applications in slow light [44, 45], optical switching [46], sensing [47], and so on.

In this work, we study the controllable optical response of a multimode optomechanical system with quadratic coupling, where two movable membranes are placed in the middle of an optical cavity with two fixed mirrors. Note that quadratic coupling has been experimentally realized in a Fabry-Pérot cavity containing a SiN membrane $[48,49]$ or a cloud of ultracold atoms [50,51], a tunable photonic crystal optomechanical cavity [52], and a microsphere-nanostring system [53]. Theoretical works have shown that quadratic coupling can be exploited to investigate mechanical squeezing [54,55], photon blockade and phonon blockade [56-59], quantum nondemolition measurement of phonons [60], quantum phase transition [61], a highly sensitive mass sensor [62], two-phonon OMIT [63], and Fano resonance [64]. Different from the linearly coupled optomechanical systems, the underlying physical mechanism in quadratically coupled optomechanical systems involves a two-phonon process [63], where the square of the displacement of the mechanical oscillator affects the response of the system. Advantages of quadratic over linear coupling include quantum nondemolition readout of a membrane's energy eigenstate [48, 49], more persistent entanglement and higher spectral nonlinearity [65], and so on. If two or more mechanical oscillators are involved, the multimode quadratic coupling optomechanical system can exhibit multiple transparency windows [66-68].

In addition, more complex interference effect occurs in optomechanical systems if the mechanical oscillator can be excited directly, which results in more interesting response property [69-76]. Zhai et al. proposed that mechanical driving field can serve as a switch of photon blockade and photon-induced tunneling [77]. In experiments, mechanical driving field has been exploited to realize electro-optomechanically induced transparency [78], cascaded optical transparency [79], phase-sensitive parametric amplifier [80], injection locking [81], and virtual exceptional points [82]. Recently, optomechanically induced opacity and amplification of two-phonon higher-order sidebands have been studied in a quadratically coupled optomechanical system with mechanical driving [83, 84]. It is pointed out that mechanical driving in the quadratically coupled system can be realized by parametrically modulating the spring constant of the membrane at twice the membrane's resonance frequency with an integrated electrical interface [80-83, 85, 86], which generates the mechanical coherence via the two-phonon process. Here we discuss the optical response properties of a multimode quadratically coupled optomechanical system to a weak probe field in the presence of a strong optical control field and two weak mechan- 
ical driving fields. We show that this system can exhibit a series of unique phenomena by tuning the optical control field and mechanical driving fields, including optomechanically induced transparency, single and double Fano resonances, and selective amplification of the weak probe field. Our results may find potential applications in optical switching based on multimode optomechanical systems.

\section{Model and theory}

We consider the optomechanical system schematically shown in Fig. 1, where two movable membranes with finite reflectivity $R_{k}(k=1,2)$ are placed in an optical cavity with two fixed mirrors. The cavity is driven by a strong control field with amplitude $\varepsilon_{c}$, frequency $\omega_{c}$, and phase $\phi_{c}$ and detected by a weak probe field with amplitude $\varepsilon_{p}$, frequency $\omega_{p}$, and phase $\phi_{p}$. Moreover, two weak coherent mechanical driving fields with amplitude $\varepsilon_{k}$, frequency $\Omega_{k}$, and phase $\phi_{k}$ are, respectively, applied to excite the two membranes. When the membranes locate at the antinodes of the intracavity standing wave, the cavity field is coupled to the square of the position of the membrane with the quadratic optomechanical coupling strength $g_{k}=\frac{8 \pi^{2} c}{\lambda^{2} L} \sqrt{\frac{R_{k}}{1-R_{k}}}$, where $c$ is the speed of light in a vacuum, $\lambda$ is the wavelength of the control field, and $L$ is the length of the cavity. The Hamiltonian of the multimode optomechanical system is given by

$$
H=\hbar \omega_{0} a^{\dagger} a+\sum_{k=1}^{2}\left(\frac{p_{k}^{2}}{2 m_{k}}+\frac{1}{2} m_{k} \omega_{k}^{2} q_{k}^{2}\right)+\sum_{k=1}^{2} \hbar g_{k} a^{\dagger} a q_{k}^{2}+H_{\mathrm{dr}},
$$

where $a^{\dagger}(a)$ is the creation (annihilation) operator of the cavity field with resonance frequency $\omega_{0}$, while $p_{k}$ and $q_{k}$ are the momentum and position operators of the $k$ th membrane with effective mass $m_{k}$ and resonance frequency $\omega_{k}$. Therefore, the first and second terms in Eq. (1) represent the energy of the cavity and mechanical modes, respectively, and the third term corresponds to the quadratic coupling between the cavity and mechanical modes. $H_{\mathrm{dr}}$ denotes the interaction between the driving fields and the optomechanical system, which takes the form

$$
\begin{aligned}
H_{\mathrm{dr}}= & i \hbar \varepsilon_{c}\left(a^{\dagger} e^{-i \omega_{c} t-i \phi_{c}}-a e^{i \omega_{c} t+i \phi_{c}}\right)+i \hbar \varepsilon_{p}\left(a^{\dagger} e^{-i \omega_{p} t-i \phi_{p}}-a e^{i \omega_{p} t+i \phi_{p}}\right) \\
& +\sum_{k=1}^{2} i \hbar \varepsilon_{k}\left[\left(b_{k}^{\dagger}\right)^{2} e^{-i \Omega_{k} t-i \phi_{k}}-b_{k}^{2} e^{i \Omega_{k} t+i \phi_{k}}\right] .
\end{aligned}
$$

The first and second terms in Eq. (2) describe the interaction between the cavity and the strong control field and the weak probe field. The amplitudes $\varepsilon_{c, p}$ are related to their re-

Figure 1 Schematic diagram of the multimode optomechanical mechanical system. The two movable membranes can be treated as mechanical modes with annihilation operators $b_{1}$ and $b_{2}$, which are quadratically coupled to the common cavity mode $a$. The cavity is driven by a control (probe) field with amplitude $\varepsilon_{c}\left(\varepsilon_{p}\right)$, frequency $\omega_{c}\left(\omega_{p}\right)$, and phase $\phi_{c}\left(\phi_{p}\right)$. In addition, the two membranes are excited by two weak coherent mechanical driving fields with amplitudes $\varepsilon_{1,2}$, frequency $\Omega=\omega_{p}-\omega_{c}$, and phases $\phi_{1,2}$

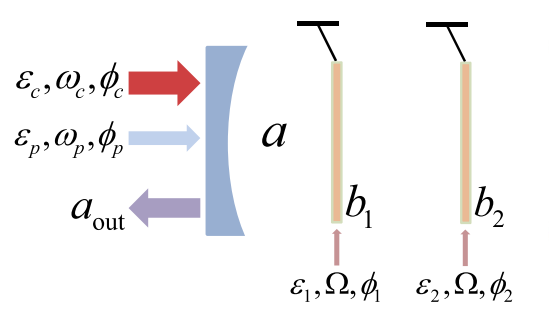


spective powers $P_{c, p}$ by the relation $\varepsilon_{c, p}=\sqrt{\kappa_{e} P_{c, p} / \hbar \omega_{c, p}}$, where $\kappa_{e}$ is the external decay rate of the cavity given by $\kappa_{e}=\eta_{c} \kappa$ with $\kappa$ being the total decay rate. The coupling parameter $\eta_{c}$ can be continuously adjusted, and we choose $\eta_{c}=0.5$ throughout this work. The last term describes the coherent mechanical driving of the two membranes. The creation (annihilation) operator $b_{k}^{\dagger}\left(b_{k}\right)$ of the membrane is defined as $b_{k}=\left(b_{k}^{\dagger}\right)^{\dagger}=\sqrt{m_{k} \omega_{k} /(2 \hbar)}\left[q_{k}+\right.$ $\left.i p_{k} /\left(m_{k} \omega_{k}\right)\right]$.

In the rotating frame at the frequency $\omega_{c}$ of the control field, the system Hamiltonian can be rewritten as

$$
\begin{aligned}
H= & \hbar \Delta_{c} a^{\dagger} a+\sum_{k=1}^{2}\left(\frac{p_{k}^{2}}{2 m_{k}}+\frac{1}{2} m_{k} \omega_{k}^{2} q_{k}^{2}\right)+\sum_{k=1}^{2} \hbar g_{k} a^{\dagger} a q_{k}^{2}+i \hbar \varepsilon_{c}\left(a^{\dagger}-a\right) \\
& +i \hbar \varepsilon_{p}\left(a^{\dagger} e^{-i \Omega t-i \phi_{p c}}-a e^{i \Omega t+i \phi_{p c}}\right) \\
& +\sum_{k=1}^{2} i \frac{m_{k} \omega_{k}}{2} \varepsilon_{k}\left[\left(q_{k}-i \frac{p_{k}}{m_{k} \omega_{k}}\right)^{2} e^{-i \Omega t-i \phi_{k}}-\left(q_{k}+i \frac{p_{k}}{m_{k} \omega_{k}}\right)^{2} e^{i \Omega t+i \phi_{k}}\right],
\end{aligned}
$$

where $\Delta_{c}=\omega_{0}-\omega_{c}, \Omega=\omega_{p}-\omega_{c}, \phi_{p c}=\phi_{p}-\phi_{c}$, and we have assumed that $\Omega_{1}=\Omega_{2}=\Omega$.

The time evolution of the system operators can be derived by applying the Heisenberg equation of motion and adding the damping and input noise terms phenomenologically, which yield

$$
\begin{aligned}
\frac{d a}{d t}= & -\left[\kappa / 2+i\left(\Delta_{c}+g_{1} q_{1}^{2}+g_{2} q_{2}^{2}\right)\right] a+\varepsilon_{c}+\varepsilon_{p} e^{-i \Omega t-i \phi_{p c}}+\sqrt{\kappa} a_{\mathrm{in}}, \\
\frac{d q_{k}}{d t}= & \frac{p_{k}}{m_{k}}+q_{k} \varepsilon_{k}\left(e^{-i \Omega t-i \phi_{k}}+e^{i \Omega t+i \phi_{k}}\right)-i \frac{p_{k}}{m_{k} \omega_{k}} \varepsilon_{k}\left(e^{-i \Omega t-i \phi_{k}}-e^{i \Omega t+i \phi_{k}}\right), \\
\frac{d p_{k}}{d t}= & -m_{k} \omega_{k}^{2} q_{k}-2 \hbar g_{k} a^{\dagger} a q_{k}-\gamma_{k} p_{k}-i m_{k} \omega_{k} q_{k} \varepsilon_{k}\left(e^{-i \Omega t-i \phi_{k}}-e^{i \Omega t+i \phi_{k}}\right) \\
& -p_{k} \varepsilon_{k}\left(e^{-i \Omega t-i \phi_{k}}+e^{i \Omega t+i \phi_{k}}\right)+\xi,
\end{aligned}
$$

where $a_{\text {in }}$ is the input vacuum noise entering the cavity with zero mean value and $\xi$ is the Brownian stochastic force acting on the membrane with zero mean value. Neglecting the weak probe field and mechanical driving field, the expectation values of the system operators at the steady state can be derived by setting the time derivatives in Eqs. (4)-(6) to zero, which are given by

$$
a_{s}=\frac{\varepsilon_{c}}{\kappa / 2+i\left(\Delta_{c}+g_{1}\left\langle q_{1}^{2}\right\rangle_{s}+g_{2}\left\langle q_{2}^{2}\right\rangle_{s}\right)}, \quad q_{k s}=0, \quad p_{k s}=0
$$

Equation (7) shows that the steady-state solutions of the momentum and position of the membranes equal to zero, and the cavity field depends on the square of the position of the membranes at the steady state, which involves a two-phonon process. Consequently, we turn to calculate the time evolution of the expectation values of the operators $a, q_{k}^{2} \equiv Q_{k}$, $p_{k}^{2} \equiv P_{k}$, and $q_{k} p_{k}+p_{k} q_{k} \equiv X_{k}$. Using the factorization assumption $\langle a b c\rangle=\langle a\rangle\langle b\rangle\langle c\rangle$ for the relevant operators, we can obtain

$$
\frac{d}{d t}\langle a\rangle=-\left[\frac{\kappa}{2}+i\left(\Delta_{c}+i g_{1}\left\langle Q_{1}\right\rangle+i g_{2}\left\langle Q_{2}\right\rangle\right)\right]\langle a\rangle+\varepsilon_{c}+\varepsilon_{p} e^{-i \Omega t-i \phi_{p c}},
$$




$$
\begin{aligned}
\frac{d}{d t}\left\langle Q_{k}\right\rangle= & \frac{1}{m_{k}}\left\langle X_{k}\right\rangle+2 \varepsilon_{k}\left(e^{-i \Omega t-i \phi_{k}}+e^{i \Omega t+i \phi_{k}}\right)\left\langle Q_{k}\right\rangle \\
& -\frac{i}{m_{k} \omega_{k}} \varepsilon_{k}\left(e^{-i \Omega t-i \phi_{k}}-e^{i \Omega t+i \phi_{k}}\right)\left\langle X_{k}\right\rangle, \\
\frac{d}{d t}\left\langle P_{k}\right\rangle= & -\left(m_{k} \omega_{k}^{2}+2 \hbar g_{k}\left\langle a^{\dagger}\right\rangle\langle a\rangle\right)\left\langle X_{k}\right\rangle-2 \gamma_{k}\left\langle P_{k}\right\rangle+\gamma_{k}\left(1+2 n_{k}\right) m_{k} \hbar \omega_{k} \\
& -2 \varepsilon_{k}\left(e^{-i \Omega t-i \phi_{k}}+e^{i \Omega t+i \phi_{k}}\right)\left\langle P_{k}\right\rangle-i m_{k} \omega_{k} \varepsilon_{k}\left(e^{-i \Omega t-i \phi_{k}}-e^{i \Omega t+i \phi_{k}}\right)\left\langle X_{k}\right\rangle, \\
\frac{d}{d t}\left\langle X_{k}\right\rangle= & \frac{2}{m_{k}}\left\langle P_{k}\right\rangle-2\left(m_{k} \omega_{k}^{2}+2 \hbar g_{k}\left\langle a^{\dagger}\right\rangle\langle a\rangle\right)\left\langle Q_{k}\right\rangle-\gamma_{k}\left\langle X_{k}\right\rangle \\
& -2 i\left(m_{k} \omega_{k}\left\langle Q_{k}\right\rangle+\frac{1}{m_{k} \omega_{k}}\left\langle P_{k}\right\rangle\right) \varepsilon_{k}\left(e^{-i \Omega t-i \phi_{k}}-e^{i \Omega t+i \phi_{k}}\right) .
\end{aligned}
$$

The term $\gamma_{k}\left(1+2 n_{k}\right) m_{k} \hbar \omega_{k}$ in Eq. (10) arises from the coupling of the membrane to the thermal environment, where $n_{k}=\left[e^{\frac{\hbar \omega_{k}}{k_{B} T}}-1\right]^{-1}$ is the mean phonon occupation number of the membrane at the temperature $T$ and $k_{B}$ is the Boltzmann's constant. In this work, both the optical probe field and the mechanical driving field are much weaker than the strong control field, thus Eqs. (8)-(11) can be solved by writing each expectation value as the sum of a steady-state solution and a small fluctuation, i.e.,

$$
\langle O\rangle=O_{s}+O_{+} e^{-i \Omega t}+O_{-} e^{i \Omega t},
$$

where $O$ represents any of these quantities $a, Q_{k}, P_{k}$, and $X_{k}$. The steady-state solutions $O_{s}$ are determined by the strong control field and are given by

$$
a_{s}=\frac{\varepsilon_{c}}{\beta}, \quad P_{k s}=\left(1+2 n_{k}\right) \frac{\hbar m_{k} \omega_{k}}{2}, \quad Q_{k s}=\frac{P_{k s}}{m_{k}^{2} \omega_{k}^{2}\left(1+\alpha_{k}\right)}, \quad X_{k s}=0
$$

with $\Delta=\Delta_{c}+g_{1} Q_{1 s}+g_{2} Q_{2 s}, \alpha_{k}=\frac{2 \hbar g_{k}\left|a_{s}\right|^{2}}{m_{k} \omega_{k}^{2}}, \beta=\kappa / 2+i \Delta$. Upon substituting Eq. (12) into Eqs. (8)-(11) and equating the coefficients of $e^{0}, e^{i \Omega t}, e^{-i \Omega t}$, we can obtain

$$
\begin{aligned}
a_{+}= & \frac{L_{1} L_{2}-i g_{1} a_{s} R_{1} L_{2}-i g_{2} a_{s} R_{2} L_{1}}{L_{1} L_{2}(\beta-i \Omega)+i g_{1} a_{s} M_{1} L_{2}+i g_{2} a_{s} M_{2} L_{1}} \varepsilon_{p} e^{-i \phi_{p c}} \\
& -\frac{i g_{1} a_{s} N_{1} L_{2}}{L_{1} L_{2}(\beta-i \Omega)+i g_{1} a_{s} M_{1} L_{2}+i g_{2} a_{s} M_{2} L_{1}} \varepsilon_{1} e^{-i \phi_{1}} \\
& -\frac{i g_{2} a_{s} N_{2} L_{1}}{L_{1} L_{2}(\beta-i \Omega)+i g_{1} a_{s} M_{1} L_{2}+i g_{2} a_{s} M_{2} L_{1}} \varepsilon_{2} e^{-i \phi_{2}},
\end{aligned}
$$

where

$$
\begin{aligned}
& L_{k}=-i \Omega m_{k}\left(\gamma_{k}-i \Omega\right)+2 m_{k} \omega_{k}^{2}\left(1+\alpha_{k}\right)-\frac{2 i \Omega m_{k} \omega_{k}^{2}\left(1+\alpha_{k}\right)}{2 \gamma_{k}-i \Omega}, \\
& M_{k}=-\frac{2 g_{k} \hbar^{2} a_{s}^{*}\left(1+2 n_{k}\right)}{m_{k} \omega_{k}\left(1+\alpha_{k}\right)}+\frac{2 g_{k} \hbar^{2} a_{s}\left(1+2 n_{k}\right)}{m_{k} \omega_{k}\left(1+\alpha_{k}\right)} \frac{\beta-i \Omega}{\beta^{*}-i \Omega}, \\
& N_{k}=\frac{\hbar\left(\gamma_{k}-i \Omega\right)\left(1+2 n_{k}\right)}{\omega_{k}\left(1+\alpha_{k}\right)}-\frac{i \hbar\left(1+2 n_{k}\right)}{1+\alpha_{k}}-i \hbar\left(1+2 n_{k}\right), \\
& R_{k}=-\frac{2 g_{k} \hbar^{2} a_{k}\left(1+2 n_{k}\right)}{\left(\beta^{*}-i \Omega\right) m_{k} \omega_{k}\left(1+\alpha_{k}\right)}
\end{aligned}
$$

with $k=1,2$. 
The output field of the optical cavity can be derived according to the input-output relation [87]

$$
a_{\text {out }}+\varepsilon_{c}+\varepsilon_{p} e^{-i \Omega t-i \phi_{p c}}=\kappa_{e}\langle a\rangle .
$$

In order to investigate the optical response of the system to the probe field, we define the corresponding quadratures of the output field oscillating at the frequency $\omega_{p}$ of the probe field as $\varepsilon_{T}=\kappa_{e} a_{+} /\left(\varepsilon_{p} e^{-i \phi_{p c}}\right)$ [69]. The real and imaginary parts of $\varepsilon_{T}$ represent the absorptive and dispersive behavior of the system to the probe field. In addition, the transmission coefficient at the frequency $\omega_{p}$ can be derived as

$$
t_{p}=\frac{\kappa_{e} a_{+}-\varepsilon_{p} e^{-i \phi_{p c}}}{\varepsilon_{p} e^{-i \phi_{p c}}}=\varepsilon_{T}-1=t_{1}+t_{2}
$$

where

$$
\begin{aligned}
t_{1}= & \frac{L_{1} L_{2}-i g_{1} a_{s} R_{1} L_{2}-i g_{2} a_{s} R_{2} L_{1}}{L_{1} L_{2}(\beta-i \Omega)+i g_{1} a_{s} M_{1} L_{2}+i g_{2} a_{s} M_{2} L_{1}} \kappa_{e}-1, \\
t_{2}= & -\frac{i g_{1} a_{s} N_{1} L_{2}}{L_{1} L_{2}(\beta-i \Omega)+i g_{1} a_{s} M_{1} L_{2}+i g_{2} a_{s} M_{2} L_{1}} \kappa_{e} r_{1} e^{-i \Phi_{1}} \\
& -\frac{i g_{2} a_{s} N_{2} L_{1}}{L_{1} L_{2}(\beta-i \Omega)+i g_{1} a_{s} M_{1} L_{2}+i g_{2} a_{s} M_{2} L_{1}} \kappa_{e} r_{2} e^{-i \Phi_{2}},
\end{aligned}
$$

with the amplitude ratio $r_{1,2}=\varepsilon_{1,2} / \varepsilon_{p}$, and phase difference $\Phi_{1,2}=\phi_{1,2}-\phi_{p c}$. Here $t_{1}$ is the contribution from the probe and control field, which results in the phenomena of OMIT and Fano resonance. The two terms in $t_{2}$ represent, respectively, the contributions from the phonon-photon processes involving the mechanical driving on the two membranes [83], which can lead to the amplification or suppression of the probe field. Interference effect between $t_{1}$ and $t_{2}$ determines the transmission (absorption) spectrum of the probe field, where the phase differences $\Phi_{1}$ and $\Phi_{2}$ play an important role.

\section{Results and discussion}

In this section, we numerically study the controllable optical response of the system using the above analytical expressions and the experimentally realizable parameters. The parameters are chosen from the recent experimental [48] and theoretical works [63]: the length of the cavity $L=6.7 \mathrm{~cm}$, and the cavity decay rate $\kappa=2 \pi \times 10^{4} \mathrm{~Hz}$; the parameters of the membranes are $\omega_{1}=\omega_{2}=\omega_{m}=2 \pi \times 10^{5} \mathrm{~Hz}, \gamma_{1}=\gamma_{2}=20 \mathrm{~Hz}, m_{1}=m_{2}=10^{-9} \mathrm{~g}$, and $R_{1}=R_{2}=0.45$. Here we have assumed that the two membranes are the same, and we will study the case that the two membranes are nondegenerate in the following. In addition, the wavelength of the control field $\lambda=\frac{2 \pi c}{\omega_{c}}=532 \mathrm{~nm}$ and the temperature of the environment $T=90 \mathrm{~K}$.

We first consider the simple case that the two membranes are the same. The phenomenon of OMIT has been observed in the probe transmission spectrum [13], and thus we plot the power transmission coefficient $\left|t_{p}\right|^{2}$ versus the normalized detuning $\Omega / \omega_{m}$ for different values of the mechanical driving fields in Fig. 2. Under the condition of twophonon resonance, i.e., $\Delta=2 \omega_{m}$, Fig. 2(a) shows that the transmission spectrum can exhibit the phenomenon of OMIT around $\Omega=2 \omega_{m}$ if $r_{1}=r_{2}=0$. The underlying mechanism of the OMIT can be explained as a result of the radiation pressure force at the beat 

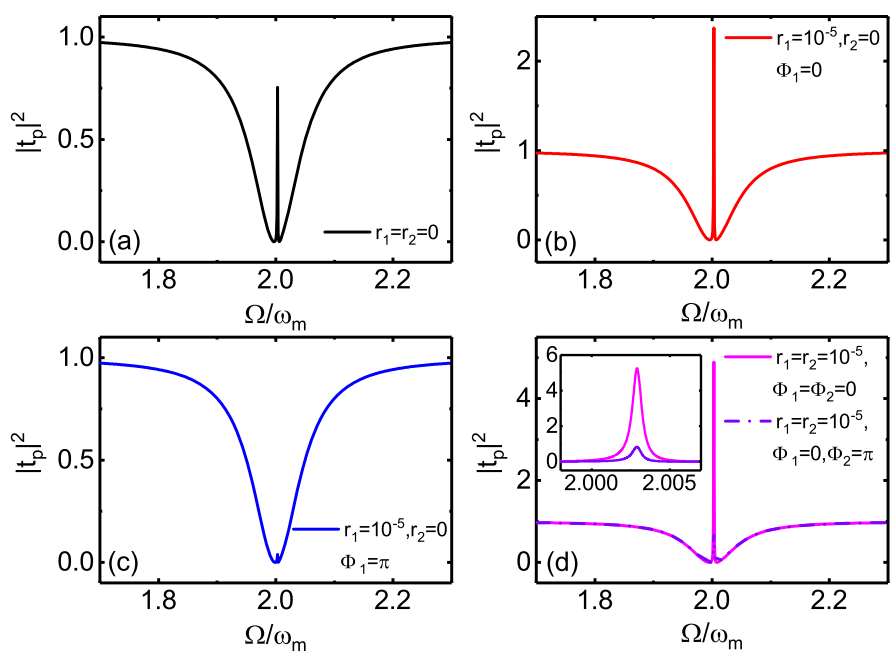

Figure 2 The power transmission coefficient $\left|t_{p}\right|^{2}$ as functions of the normalized detuning $\Omega / \omega_{m}$ for different values of the mechanical driving fields. Other parameters are $\lambda=532 \mathrm{~nm}, L=6.7 \mathrm{~cm}$, $\kappa=2 \pi \times 10^{4} \mathrm{~Hz}, \eta_{c}=0.5, \omega_{1}=\omega_{2}=\omega_{m}=2 \pi \times 10^{5} \mathrm{~Hz}, \gamma_{1}=\gamma_{2}=20 \mathrm{~Hz}, m_{1}=m_{2}=10^{-9} \mathrm{~g}, R_{1}=R_{2}=0.45$, $T=90 \mathrm{~K}, P_{c}=90 \mu \mathrm{W}$, and $\Delta=2 \omega_{m}$

frequency $\Omega$ between the probe and control photons. The membranes can vibrate coherently under the action of the radiation pressure, which in turn generates the Stokes-and anti-Stokes scattering of light from the strong control field via the two-phonon process. At $\Delta=2 \omega_{m}$, the highly off-resonant Stokes scattering at frequency $\omega_{c}-2 \omega_{m}$ is suppressed and only anti-Stokes scattering at frequency $\omega_{c}+2 \omega_{m}$ builds up inside the cavity. However, if the incident probe field is nearly resonant with the cavity field, destructive interference between the probe field and the anti-Stokes field can suppress the build-up of an intracavity probe field, which results in a transparency window in the transmission spectrum. Such a two-phonon OMIT has been extensively investigated in recent works by discussing the absorption $\operatorname{Re}\left(\varepsilon_{T}\right)[63,66,67]$. Moreover, the transmission spectrum can be further modified by the additional mechanical driving fields. If only one membrane is excited by a coherent mechanical driving field $\left(r_{1}=10^{-5}\right)$, the transparency window in Fig. 2(a) becomes a transmission peak with $\left|t_{p}\right|^{2} \approx 2.4$ for $\Phi_{1}=0$, as shown in Fig. 2(b). Therefore, the weak probe field can be amplified due to the additional mechanical driving field, which can be explained by the interference effect as follows. In the simultaneous presence of a strong control field, a weak probe field, and a weak coherent mechanical driving field, the energy level of the system can form a closed-loop transition structure, giving rise to the phase-dependent optical response properties [69-75]. At $\Phi_{1}=0$, constructive interference between $t_{1}$ and the first term in $t_{2}$ results in the amplification of the probe field [75]. If the phase difference $\Phi_{1}$ is tuned to be $\pi$, Fig. 2(c) shows that destructive interference between $t_{1}$ and $t_{2}$ results in the strong suppression of transmission with $\left|t_{p}\right|^{2} \approx 0.04$ around $\Omega / \omega_{m}=2$. The interference effect in this system becomes more complicated when both the membranes are excited directly. At $r_{1}=r_{2}=10^{-5}$ and $\Phi_{1}=\Phi_{2}=0$, the peak transmission coefficient at $\Omega / \omega_{m} \approx 2.0029$ is further enhanced to be $\left|t_{p}\right|^{2} \approx 5.3$ because the two terms in $t_{2}$ interfere constructively. If $\Phi_{1}=0$ but $\Phi_{2}=\pi$, the two terms in $t_{2}$ interfere destructively, and the inset of Fig. 2(d) shows that the peak transmission coefficient around $\Omega / \omega_{m}=2$ is almost equal to that in Fig. 2(a). Consequently, the optical response of this 
Figure 3 Contour plot of the power transmission coefficient $\left|t_{p}\right|^{2}$ at $\Omega=2.0029 \omega_{m}$ versus the phase difference $\Phi_{1} / \pi$ and $\Phi_{2} / \pi$. The other parameters are the same as those in Fig. 2 except $r_{1}=r_{2}=10^{-5}$

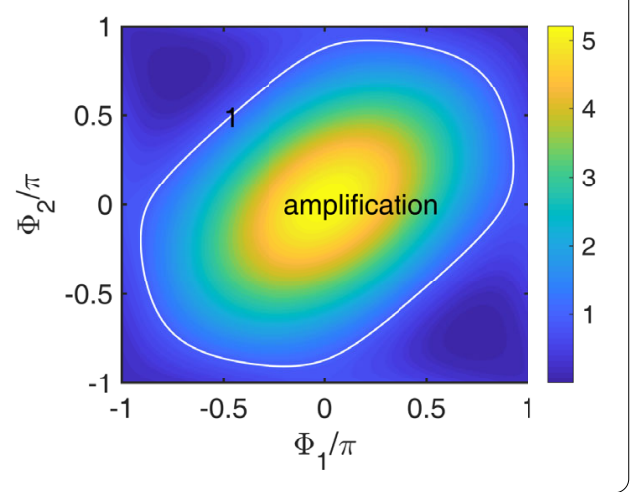

system can be controlled more flexibly when the mechanical driving field is modulated independently.

In order to see the effect of phase difference more clearly, we plot the power transmission coefficient $\left|t_{p}\right|^{2}$ at $\Omega=2.0029 \omega_{m}$ as functions of $\Phi_{1} / \pi$ and $\Phi_{2} / \pi$ in Fig. 3. It is shown that the transmission coefficient $\left|t_{p}\right|^{2}$ reaches the maximum around $\Phi_{1}=\Phi_{2}=0$ with peak value $\left|t_{p}\right|^{2} \approx 5.3$, and the minimum value is obtained around $\left(\Phi_{1}=-0.75 \pi, \Phi_{2}=0.75 \pi\right)$ and $\left(\Phi_{1}=0.75 \pi, \Phi_{2}=-0.75 \pi\right)$ with $\left|t_{p}\right|^{2} \approx 0$. This phase dependent phenomenon arises from the interference effect in that we consider $\omega_{1}=\omega_{2}=\omega_{m}, \Omega_{1}=\Omega_{2}=\Omega$ and $r_{1}=r_{2}$ here. Moreover, the contour line with $\left|t_{p}\right|^{2}=1$ forms a "circular runway". The transmitted probe field can be amplified inside the contour line, otherwise it will be attenuated.

We have shown that a symmetric peak locates around $\Omega / \omega_{m}=2$ in the transmission spectrum under the two-phonon resonance condition. If the cavity-control field detuning $\Delta \neq 2 \omega_{m}$, asymmetric Fano line shape can be observed [64, 68]. Similar to previous works about Fano resonance in optomechanical systems [22, 23, 64, 68, 76], we also study the absorptive behavior $\operatorname{Re}\left(\varepsilon_{T}\right)$ of the output probe field. At small coupling parameter $\eta_{c} \ll 1$, we can obtain $\left|t_{p}\right| \simeq 1-\operatorname{Re}\left(\varepsilon_{T}\right)$ and $\arg \left(t_{p}\right) \simeq-\operatorname{Im}\left(\varepsilon_{T}\right)$ [69]. Therefore, both the transmission $\left|t_{p}\right|^{2}$ and absorption $\operatorname{Re}\left(\varepsilon_{T}\right)$ can reveal the same phenomena of the system. At $\Delta=1.9 \omega_{m}$, Fig. 4 plots the absorption $\operatorname{Re}\left(\varepsilon_{T}\right)$ of the output probe field versus the normalized detuning $\Omega / \omega_{m}$ when one membrane is excited with different phases. In the absence of the mechanical driving field, the top panel in Fig. 4 shows that the absorption spectrum can exhibit an asymmetric Fano line shape around $\Omega / \omega_{m}=2$ and a broad absorption peak around $\Omega / \omega_{m}=1.9$. The asymmetric Fano line shape results from the destructive interference between the anti-Stokes field and the probe field at frequency $\omega_{p}=\omega_{c}+2 \omega_{m}$, where the anti-Stokes field is not resonant with the cavity frequency $\omega_{0}$. The broad absorption peak at $\Omega / \omega_{m}=1.9$ is due to the resonant absorption of the probe photons by the cavity. When the mechanical driving field is turned on, the absorption spectrum can be modified, depending on the phase difference. At $r_{1}=10^{-5}$ and $\Phi_{1}=0$, the minimum absorption $\operatorname{Re}\left(\varepsilon_{T}\right)$ in the vicinity of $\Omega / \omega_{2}=2$ is negative, which indicates the amplification of the probe field. A transition between amplification and absorption occurs when the normalized detuning $\Omega / \omega_{m}$ increases. At fixed amplitude ratio $r_{1}$, Fig. 4 shows that the asymmetric Fano line shape around $\Omega / \omega_{m}=2$ can be modulated effectively for various phase difference $\Phi_{1}$, where the interference effect is evident. However, the absorption spectrum in other parameter regime almost keeps the same.

We have assumed that the resonance frequencies of the two membranes are the same in the above, but it is possible to tune the resonance frequency independently, which en- 


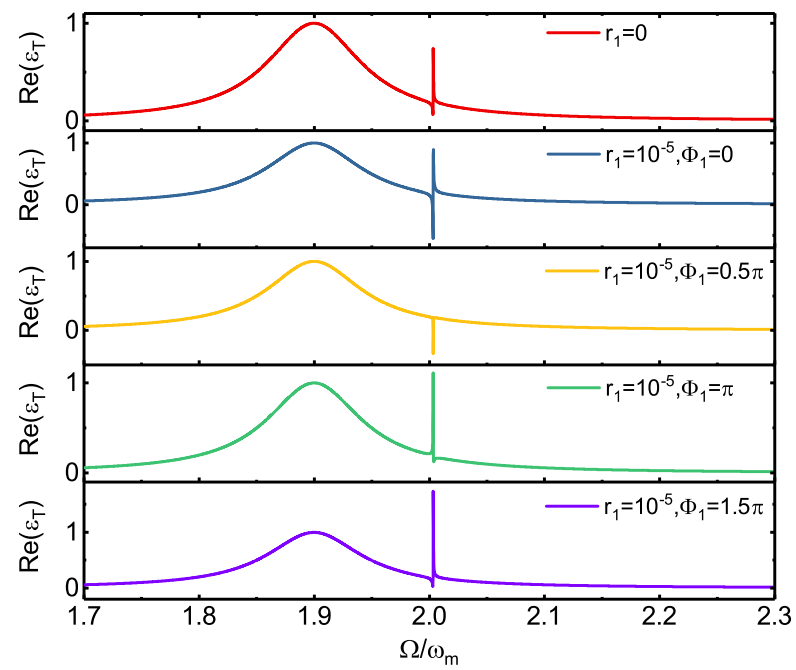

Figure 4 The absorption $\operatorname{Re}\left(\varepsilon_{T}\right)$ of the output probe field as a function of the normalized detuning $\Omega / \omega_{m}$ for different values of the mechanical driving field. The other parameters are the same as those in Fig. 2 except $\Delta=1.9 \omega_{m}$ and $r_{2}=0$
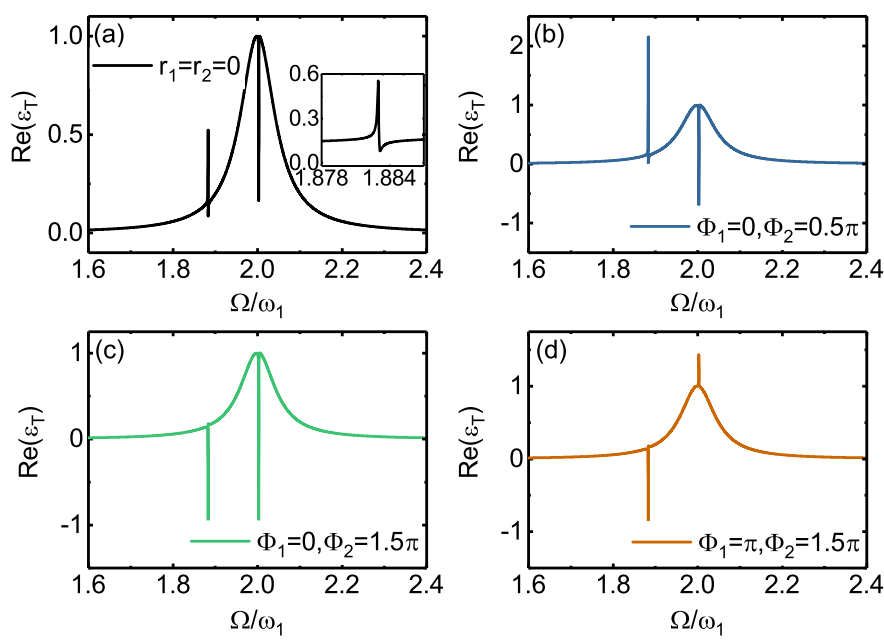

Figure 5 The absorption $\operatorname{Re}\left(\varepsilon_{T}\right)$ of the output probe field versus the normalized detuning $\Omega / \omega_{1}$ for different values of the mechanical driving fields. The other parameters are the same as those in Fig. 2 except $\omega_{1}=2 \pi \times 10^{5} \mathrm{~Hz}, \omega_{2}=2 \pi \times 0.94 \times 10^{5} \mathrm{~Hz}$, and $\Delta=2 \omega_{1}$. In Figs. $5(\mathbf{c})-(\mathbf{d})$, we choose $r_{1}=r_{2}=10^{-5}$

ables us to control the optical response of the system more flexibly. For $\omega_{1}=2 \pi \times 10^{5} \mathrm{~Hz}$ and $\omega_{2}=2 \pi \times 0.94 \times 10^{5} \mathrm{~Hz}$, we plot the absorption $\operatorname{Re}\left(\varepsilon_{T}\right)$ of the output probe field as a function of the normalized detuning $\Omega / \omega_{1}$ in Fig. 5 with $\Delta=2 \omega_{1}$. In this case, the condition of two-phonon resonance is only satisfied for the membrane with resonance frequency $\omega_{1}$. In the absence of the mechanical driving field, the absorption spectrum in Fig. 5(a) exhibits a symmetric absorption dip around $\Omega / \omega_{1}=2$, which indicates the appearance of optomechanically induced transparency (OMIT), and an asymmetric Fano line shape near $\Omega / \omega_{1}=2 \omega_{2} / \omega_{1}=1.88$. OMIT and Fano line shape result from the destructive interference between the probe field and the generated anti-Stokes fields at fre- 
quency $\omega_{c}+2 \omega_{1}$ and $\omega_{c}+2 \omega_{2}$, respectively. When both the mechanical driving fields are switched on with fixed amplitudes and various phases, the absorption spectra can be modified, as shown in Figs. 5(b)-5(d). At $\Phi_{1}=0$ and $\Phi_{2}=0.5 \pi$, the absorption peak around $\Omega / \omega_{1}=1.88$ becomes larger than 1 , which indicates the enhanced absorption due to the mechanical driving field. However, the absorption dip around $\Omega / \omega_{1}=2$ becomes negative, which corresponds to the amplification of the probe field. By tuning the phase differences $\Phi_{1}$ and $\Phi_{2}$ independently, we can see from Figs. 5(c)-5(d) that the two resonances around $\Omega / \omega_{1}=1.88$ and $\Omega / \omega_{1}=2$ switch between the enhanced absorption and amplification. Therefore, the output probe field can be selectively amplified by tuning the mechanical driving fields. Different from the case that $\omega_{1}=\omega_{2}=\omega_{m}$, Fig. 5 demonstrates that the absorption curves around $\Omega / \omega_{1}=1.88$ and $\Omega / \omega_{1}=2$ are controlled independently by tuning the phase differences. The two mechanical driving fields cannot interfere with each other since the frequency difference $\left|\omega_{1}-\omega_{2}\right|$ is much larger than the linewidth of the absorption peaks (dips) around $\Omega / \omega_{1}=1.88$ and $\Omega / \omega_{1}=2$.

When the cavity-control field detuning is tuned to be $\Delta=\omega_{1}+\omega_{2}$ with $\omega_{1}=2 \pi \times 10^{5} \mathrm{~Hz}$ and $\omega_{2}=2 \pi \times 0.8 \times 10^{5} \mathrm{~Hz}$, the absorption $\operatorname{Re}\left(\varepsilon_{T}\right)$ of the output probe field exhibits a broad absorption peak in the center and two sideband peaks (dips). Figures 6(b) and 6(c) are the enlargement of the two sideband peaks around $\Omega / \omega_{1}=1.6$ and $\Omega / \omega_{1}=2$, in which the red solid curves correspond to the asymmetric Fano line shapes for $r_{1}=r_{2}=0$. In this case, both the generated anti-Stokes fields at frequencies $\omega_{c}+2 \omega_{1}$ and $\omega_{c}+2 \omega_{2}$ are not resonant with the cavity frequency. The Fano resonance around $\Omega / \omega_{1}=2$ is caused by interference effect between the probe field and the anti-Stokes field at frequency $\omega_{c}+2 \omega_{1}$, while the Fano resonance around $\Omega / \omega_{1}=1.6$ is due to the interference effect at frequency $\omega_{c}+2 \omega_{2}$. Therefore, the phenomena of a single OMIT and a single Fano resonance in Fig. 5 can be switched to double Fano resonances by modulating the cavity-control field
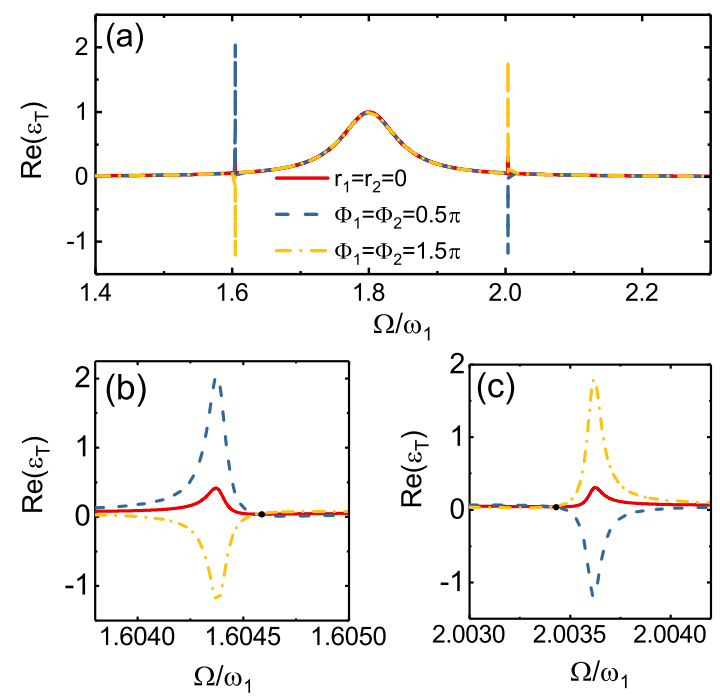

Figure 6 The absorption $\operatorname{Re}\left(\varepsilon_{T}\right)$ of the output probe field as a function of the normalized detuning $\Omega / \omega_{1}$ for different values of the mechanical driving fields. Figure $6(\mathbf{b})$ and $6(\mathbf{c})$ are the enlarged images of Fig. $6(\mathbf{a})$ around $\Omega / \omega_{1}=1.6$ and $\Omega / \omega_{1}=2.0$, respectively. The other parameters are the same as those in Fig. 5 except $\omega_{2}=2 \pi \times 0.8 \times 10^{5} \mathrm{~Hz}$ and $\Delta=2 \omega_{m}=\omega_{1}+\omega_{2}$. The dashed and dash-dotted curves correspond to $r_{1}=r_{2}=10^{-5}$ 
(a)

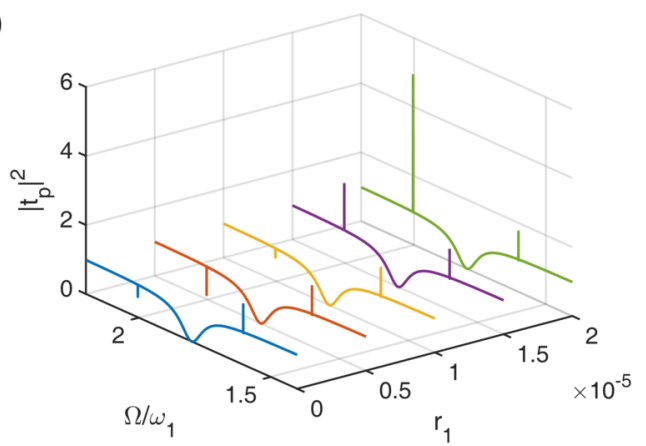

(b)

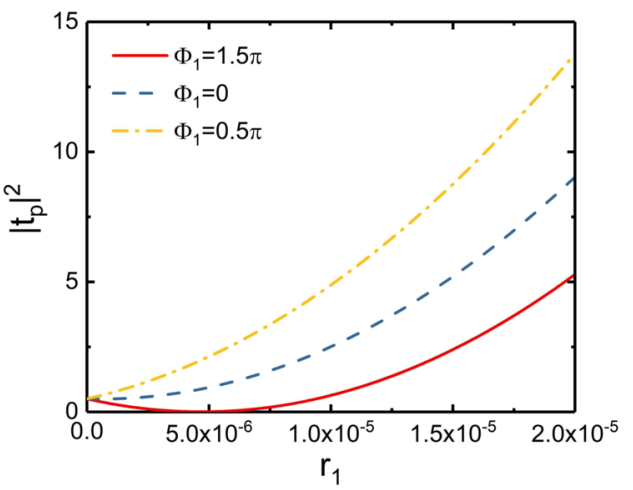

Figure 7 (a) The power transmission coefficient $\left|t_{p}\right|^{2}$ as functions of the normalized detuning $\Omega / \omega_{1}$ for different values of the amplitude ratio $r_{1}$ with $\Phi_{1}=1.5 \pi$. (b) The transmission coefficient $\left|t_{p}\right|^{2}$ at $\Omega=2.00362 \omega_{1}$ versus the amplitude ratio $r_{1}$ with $\Phi_{1}=1.5 \pi, 0$, and $0.5 \pi$, respectively. The other parameters are the same as those in Fig. 6 except $r_{2}=10^{-5}$ and $\Phi_{2}=0.5 \pi$

detuning $\Delta$. Moreover, the Fano line shapes can be modified by the mechanical driving fields. At $\Phi_{1}=\Phi_{2}=0.5 \pi$, the peak value around $\Omega / \omega_{1}=1.6$ becomes larger than 1 that is an indication of enhanced absorption, but the absorption peak near $\Omega / \omega_{1}=2$ becomes an absorption dip with negative value of $\operatorname{Re}\left(\varepsilon_{T}\right)$. The double Fano resonance is reversed if $\Phi_{1}=\Phi_{2}=1.5 \pi$ compared with $\Phi_{1}=\Phi_{2}=0.5 \pi$.

Finally, we study the effect of the amplitude of the mechanical driving field on the transmission spectrum. Figure 7(a) plots the power transmission coefficient $\left|t_{p}\right|^{2}$ versus the normalized detuning $\Omega / \omega_{1}$ for $r_{1}=0,0.5 \times 10^{-5}, 1.0 \times 10^{-5}, 1.5 \times 10^{-5}$, and $2 \times 10^{-5}$, respectively. Here we keep $\Phi_{1}=1.5 \pi, r_{2}=10^{-5}, \Phi_{2}=0.5 \pi$ fixed. For $r_{1}=0$, the transmission coefficient $\left|t_{p}\right|^{2}<1$ at $\Omega / \omega_{1} \approx 2$ but $\left|t_{p}\right|^{2}>1$ at $\Omega / \omega_{1} \approx 1$.6. For $r_{1}=0.5 \times 10^{-5}$, the minimum value of $\left|t_{p}\right|^{2}$ becomes smaller due to the interference effect induced by the mechanical driving field. When the amplitude ratio $r_{1}$ is increased to $10^{-5}$, the minimum transmission coefficient $\left|t_{p}\right|^{2}$ near $\Omega / \omega_{1}=2$ becomes larger. At higher value of $r_{1}$, the transmission dip is switched to a transmission peak with $\left|t_{p}\right|^{2}>1$. Meanwhile, the transmission peak around $\Omega / \omega_{1} \approx 1.6$, which is determined by the interference effect at frequency $\omega_{c}+2 \omega_{2}$, keeps almost the same when the amplitude ratio $r_{1}$ increases. In Fig. 7(b), the transmission coefficient $\left|t_{p}\right|^{2}$ at $\Omega=2.00362 \omega_{1}$ is plotted as a function of the amplitude ratio $r_{1}$ for various values of phase difference $\Phi_{1}$. At $\Phi_{1}=1.5 \pi$, the transmission coefficient $\left|t_{p}\right|^{2}$ decreases from an initial value to zero when the amplitude ratio $r_{1}$ increases. With further increasing the amplitude ratio $r_{1}$, the transmission coefficient $\left|t_{p}\right|^{2}$ starts to increase again and can be larger than 1 . This phenomenon can be well explained in terms of the 
complicated interference effect induced by the mechanical driving field [75]. In addition, the phase-dependent effect can be seen from the curves for $\Phi_{1}=0$ and $\Phi_{1}=0.5 \pi$, where the transmission coefficient $\left|t_{p}\right|^{2}$ increases monotonically with the enhancement of the amplitude ratio $r_{1}$.

\section{Conclusion}

In conclusion, we have studied the controllable optical response of a multimode optomechanical system with quadratic coupling, where two movable membranes are placed in an optical cavity with two fixed mirrors. The response of the system to a weak probe field is investigated when the cavity is driven by a strong control field and the membranes are, respectively, excited by weak coherent mechanical driving fields. If the two membranes have the same resonance frequency, a single optomechanically induced transparency window occurs in the transmission spectrum under the condition of two-phonon resonance, which can be further modified by the two mechanical driving fields. When the condition of two-phonon resonance is not satisfied, the absorption spectrum can exhibit a single asymmetric Fano line shape. It is shown that the switch between the amplification and enhanced absorption of the probe field can be realized by tuning the phases of the mechanical driving fields. If the frequencies of the two membranes are different, by tuning the cavity-control field detuning, the absorption spectrum can exhibit the phenomenon of a single OMIT and a single Fano line shape or the phenomenon of double Fano line shapes, which results from the interference effect between the probe field and the two generated anti-Stokes fields. Moreover, the line shapes around the two frequencies of the anti-Stokes fields can be controlled independently by the phases and amplitudes of the two mechanical driving fields.

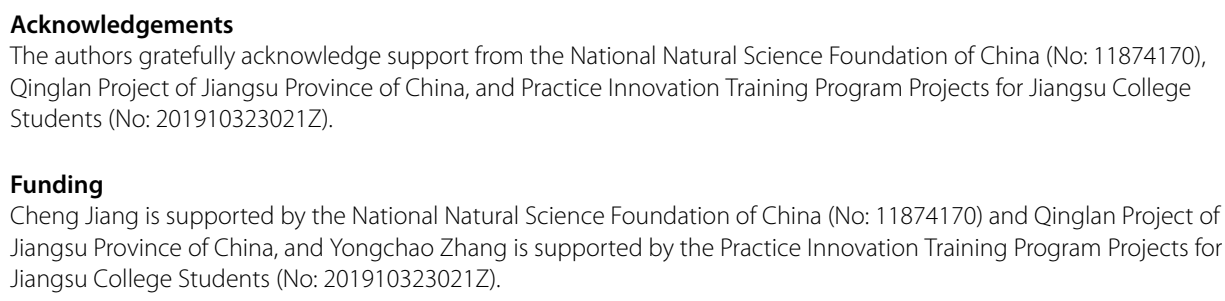

Abbreviations

OMIT, Optomechanically induced transparency; OMIA, Optomechanically induced amplification; EIT, Electromagnetically induced transparency.

Availability of data and materials

Not applicable. For all requests relating to the paper, please contact the author.

Competing interests

The authors declare that they have no competing interests.

Authors' contributions

CJ finished the main work of the paper, including conceiving of the idea, plotting the figures, and revising the draft. $Y Z$ deduced the main formulas of the paper and drafted the manuscript. XC revised the draft and discussed the main results of the paper. All authors read and approved the final manuscript.

\section{Publisher's Note}

Springer Nature remains neutral with regard to jurisdictional claims in published maps and institutional affiliations. 


\section{References}

1. Marquardt F, Girvin SM. Optomechanics. Physics. 2009;2:40.

2. Aspelmeyer M, Kippenberg TJ, Marquardt F. Cavity optomechanics. Rev Mod Phys. 2014;86(4):1391-452.

3. Xiong H, Si LG, Lv XY et al. Review of cavity optomechanics in the weak-coupling regime: from linearization to intrinsic nonlinear interactions. Sci China, Phys Mech Astron. 2015;58(5):1-13.

4. Teufel JD, Donner T, Li D et al. Sideband cooling of micromechanical motion to the quantum ground state. Nature. 2011;475:359-63.

5. Chan J, Mayer Alegre TP, Safavi-Naeini AH et al. Laser cooling of a nanomechanical oscillator into its quantum ground state. Nature. 2011;478:89-92.

6. Safavi-Naeini AH, Gröblacher S, Hill JT et al. Squeezed light from a silicon micromechanical resonator. Nature. 2013:500:185-9.

7. Purdy TP, Yu PL, Peterson RW et al. Strong optomechanical squeezing of light. Phys Rev X. 2013;3:031012.

8. Wollman EE, Lei CU, Weinstein AJ et al. Quantum squeezing of motion in a mechanical resonator. Science. 2015;349:952-5

9. Pirkkalainen JM, Damskägg E, Brandt M et al. Squeezing of quantum noise of motion in a micromechanical resonator. Phys Rev Lett. 2015;115:243601.

10. Ockeloen-Korppi CF, Damskägg E, Pirkkalainen JM et al. Stabilized entanglement of massive mechanical oscillators. Nature. 2018;556:478-82.

11. Riedinger R, Wallucks A, Marinković I et al. Remote quantum entanglement between two micromechanical oscillators. Nature. 2018;556:473-7.

12. Agarwal GS, Huang SM. Electromagnetically induced transparency in mechanical effects of light. Phys Rev A. 2010;81:041803.

13. Weis S, Rivière R, Deléglise S et al. Optomechanically induced transparency. Science. 2010;330(6010):1520-3.

14. Safavi-Naeini AH, Mayer Alegre TP, Chan J et al. Electromagnetically induced transparency and slow light with optomechanics. Nature. 2011;472:69-73.

15. Xiong H, Wu Y. Fundamentals and applications of optomechanically induced transparency. Appl Phys Rev. 2018;5:031305

16. Chen HJ. Optomechanically induced transparency and nonlinear responses based on graphene optomechanics system. EPJ Quantum Technol. 2019;6:3.

17. Karuza M, Biancofiore C, Bawaj M et al. Optomechanically induced transparency in a membrane-in-the-middle setup at room temperature. Phys Rev A. 2013:88:013804.

18. Burek MJ, Cohen JD, Meenehan SM et al. Diamond optomechanical crystals. Optica. 2016;3(12):1404-11.

19. Shen Z, Dong $\mathrm{CH}$, Chen $\mathrm{Y}$ et al. Compensation of the Kerr effect for transient optomechanically induced transparency in a silica microsphere. Opt Lett. 2016;41(6):1249-52.

20. Massel F, Heikkilä TT, Pirkkalainen JM et al. Microwave amplification with nanomechanical resonators. Nature. 2011:480:351-4

21. Singh V, Bosman SJ, Schneider BH et al. Optomechanical coupling between a multilayer graphene mechanical resonator and a superconducting microwave cavity. Nat Nanotechnol. 2014;9:820-4.

22. Qu KN, Agarwal GS. Fano resonances and their control in optomechanics. Phys Rev A. 2013;87:063813.

23. Jiang $C$, Jiang $L, Y u H L$ et al. Fano resonance and slow light in hybrid optomechanics mediated by a two-level system. Phys Rev A. 2017;96:053821.

24. Fleischhauer M, Imamoglu A, Marangos JP. Electromagnetically induced transparency: optics in coherent media. Rev Mod Phys. 2005;77:633.

25. Wu Y, Yang XX. Electromagnetically induced transparency in $V$-, $\Lambda$-, and cascade-type schemes beyond steady-state analysis. Phys Rev A. 2005;71:053806.

26. Boller KJ, Imamoğlu A, Harris SE. Observation of electromagnetically induced transparency. Phys Rev Lett. 1991:66:2593.

27. Phillips MC, Wang H, Rumyantsev I et al. Electromagnetically induced transparency in semiconductors via biexciton coherence. Phys Rev Lett. 2003:91:183602.

28. Liu N, Langguth L, Weiss T et al. Plasmonic analogue of electromagnetically induced transparency at the Drude damping limit. Nat Mater. 2009;8:758-62.

29. Peng B, Özdemir ŞK, Chen WJ et al. What is and what is not electromagnetically induced transparency in whispering-gallery microcavities. Nat Commun. 2014;5:5082

30. Xiong H, Si LG, Zheng AS et al. Higher-order sidebands in optomechanically induced transparency. Phys Rev A. 2012:86:013815.

31. Jiao Y, Lü H, Qian J et al. Nonlinear optomechanics with gain and loss: amplifying higher-order sideband and group delay. New J Phys. 2016;18:083034.

32. Jiao YF, Lu TX, Jing H. Optomechanical second-order sidebands and group delays in a Kerr resonator. Phys Rev A 2018;97:013843.

33. Wang H, Gu X, Liu Y et al. Optomechanical analog of two-color electromagnetically induced transparency: photon transmission through an optomechanical device with a two-level system. Phys Rev A. 2014;90:023817.

34. Xiong H, Huang YM, Wan LL et al. Vector cavity optomechanics in the parameter configuration of optomechanically induced transparency. Phys Rev A. 2016;94:013816

35. Jing H, Özdemir ŞK, Geng Z et al. Optomechanically-induced transparency in parity-time-symmetric microresonators. Sci Rep. 2015;5:9663.

36. Lü H, Wang CQ, Yang L et al. Optomechanically induced transparency at exceptional points. Phys Rev Appl. 2018:10:014006.

37. Zhang H, Saif F, Jiao $Y$ et al. Loss-induced transparency in optomechanics. Opt Express. 2018;26:25199.

38. Shen Z, Zhang YL, Chen Y et al. Experimental realization of optomechanically induced non-reciprocity. Nat Photonics. 2016;10:657-61.

39. Lü H, Jiang Y, Wang YZ et al. Optomechanically induced transparency in a spinning resonator. Photon Res. 2017;5:367-71. 
40. Huang R, Miranowicz A, Liao JQ et al. Nonreciprocal photon blockade. Phys Rev Lett. 2018;121:153601.

41. Jiao YF, Zhang SD, Zhang YL et al. Nonreciprocal optomechanical entanglement against backscattering losses. Phys Rev Lett. 2020;125:143605.

42. Fano U. Effects of configuration interaction on intensities and phase shifts. Phys Rev. 1961:124:1866.

43. Miroshnichenko AE, Flach S, Kivshar YS. Fano resonances in nanoscale structures. Rev Mod Phys. 2010;82:2257.

44. Hau LV, Harris SE, Dutton Z et al. Light speed reduction to 17 metres per second in an ultracold atomic gas. Nature. 1999;397:594.

45. Wu C, Khanikaev AB, Shvets G. Broadband slow light metamaterial based on a double-continuum Fano resonance. Phys Rev Lett. 2011;106:107403.

46. Stern L, Grajower M, Levy U. Fano resonances and all-optical switching in a resonantly coupled plasmonic-atomic system. Nat Commun. 2014:5:4865

47. Wu C, Khanikaev AB, Adato R et al. Fano-resonant asymmetric metamaterials for ultrasensitive spectroscopy and identification of molecular monolayers. Nat Mater. 2012;11:69.

48. Thompson JD, Zwickl BM, Jayich AM et al. Strong dispersive coupling of a high-finesse cavity to a micromechanical membrane. Nature. 2008;452:72-5.

49. Sankey JC, Yang C, Zwickl BM et al. Strong and tunable nonlinear optomechanical coupling in a low-loss system. Nat Phys. 2010;6:707-12.

50. Murch KW, Moore KL, Gupta S et al. Observation of quantum-measurement backaction with an ultracold atomic gas. Nat Phys. 2008;4:561-4

51. Purdy TP, Brooks DWC, Botter T et al. Tunable cavity optomechanics with ultracold atoms. Phys Rev Lett. 2010;105:133602.

52. Paraïso TK, Kalaee M, Zang L et al. Position-squared coupling in a tunable photonic crystal optomechanical cavity. Phys Rev X. 2015;5:041024

53. Brawley GA, Vanner MR, Larsen PE et al. Nonlinear optomechanical measurement of mechanical motion. Nat Commun. 2016:7:10988.

54. Nunnenkamp A, Børkje K, Harris JGE et al. Cooling and squeezing via quadratic optomechanical coupling. Phys Rev A. 2010;82:021806

55. Asjad M, Agarwal GS, Kim MS et al. Robust stationary mechanical squeezing in a kicked quadratic optomechanical system. Phys Rev A. 2014;89:023849.

56. Liao JQ, Nori F. Photon blockade in quadratically coupled optomechanical systems. Phys Rev A. 2013;88:023853.

57. Xie H, Liao CG, Shang X et al. Phonon blockade in a quadratically coupled optomechanical system. Phys Rev A. 2017:96:013861.

58. Xu XW, Shi HQ, Chen AX et al. Cross-correlation between photons and phonons in quadratically coupled optomechanical systems. Phys Rev A. 2018;98:013821.

59. Zhang JS, Li MC, Chen AX. Enhancing quadratic optomechanical coupling via a nonlinear medium and lasers. Phys Rev A. 2019;99:013843.

60. Hauer BD, Metelmann A, Davis JP. Phonon quantum nondemolition measurements in nonlinearly coupled optomechanical cavities. Phys Rev A. 2018;98:043804.

61. Lü XY, Zheng LL, Zhu GL et al. Single-photon-triggered quantum phase transition. Phys Rev Appl. 2018:9:064006

62. Liu S, Liu B, Wang J et al. Realization of a highly sensitive mass sensor in a quadratically coupled optomechanical system. Phys Rev A. 2019;99:033822.

63. Huang S, Agarwal GS. Electromagnetically induced transparency from two-phonon processes in quadratically coupled membranes. Phys Rev A. 2011;83:023823.

64. Huang S, Chen A. Fano resonance and amplification in a quadratically coupled optomechanical system with a Kerr medium. Phys Rev A. 2020;101:023841.

65. Shi H, Bhattacharya M. Quantum mechanical study of a generic quadratically coupled optomechanical system. Phys Rev A. 2013:87:043829.

66. Xiao RJ, Pan GX, Zhou L. Analog multicolor electromagnetically induced transparency in multimode quadratic coupling quantum optomechanics. J Opt Soc Am B. 2015;32(7):1399-405.

67. He Q, Badshah F, Din RU et al. Multiple transparency in a multimode quadratic coupling optomechanical system with an ensemble of three-level atoms. J Opt Soc Am B. 2018;35(10):2550-61.

68. Wang XY, Si LG, Lu XH et al. Optomechanically tuned Fano resonance and slow light in a quadratically coupled optomechanical system with membranes. J Phys B, At Mol Opt Phys. 2020;53:235402.

69. Jia WZ, Wei LF, Li Y et al. Phase-dependent optical response properties in an optomechanical system by coherently driving the mechanical resonator. Phys Rev A. 2015;91:043843.

70. Ma JY, You C, Si LG et al. Optomechanically induced transparency in the presence of an external time-harmonic-driving force. Sci Rep. 2015;5:11278.

71. Xu XW, Li Y. Controllable optical output fields from an optomechanical system with mechanical driving. Phys Rev A 2015;92:023855

72. Li Y, Huang YY, Zhang XZ et al. Optical directional amplification in a three-mode optomechanical system. Opt Express. 2017;25:18907-16

73. Lu TX, Jiao YF, Zhang HL et al. Selective and switchable optical amplification with mechanical driven oscillators. Phys Rev A. 2019:100:013813.

74. Jiang C, Cui YS, Zhai ZY et al. Phase-controlled amplification and slow light in a hybrid optomechanical system. Opt Express. 2019:27:30473-85.

75. Zhang Y, Yan K, Zhai Z et al. Mechanical driving mediated slow light in a quadratically coupled optomechanical system. J Opt Soc Am B. 2020;37(3):650-7.

76. Pramanik N, Yellapragada KC, Singh S et al. Coherent control of Fano resonances in a macroscopic four-mirror cavity. Phys Rev A. 2020;101:043802.

77. Zhai C, Huang $\mathrm{R}$, Jing $\mathrm{H}$ et al. Mechanical switch of photon blockade and photon-induced tunneling. Opt Express. 2019;27(20):27649-62.

78. Bochmann J, Vainsencher A, Awschalom DD et al. Nanomechanical coupling between microwave and optical photons. Nat Phys. 2013;9:712-6. 
79. Fan L, Fong KY, Poot M et al. Cascaded optical transparency in multimode-cavity optomechanical systems. Nat Commun. 2015;6:5850.

80. Bothner D, Yanai S, Iniguez-Rabago A et al. Cavity electromechanics with parametric mechanical driving. Nat Commun. 2020:11:1589.

81. Bekker C, Kalra R, Baker C et al. Injection locking of an electro-optomechanical device. Optica. 2017;4(10):1 196-204.

82. Renault P, Yamaguchi H, Mahboob I. Virtual exceptional points in an electromechanical system. Phys Rev Appl. 2019;11(2):024007.

83. Si LG, Xiong H, Zubairy MS et al. Optomechanically induced opacity and amplification in a quadratically coupled optomechanical system. Phys Rev A. 2017;95:033803.

84. Liu SP, Yang WX, Shui T et al. Tunable two-phonon higher-order sideband amplification in a quadratically coupled optomechanical system. Sci Rep. 2017;7:17637.

85. Rugar D, Grütter P. Mechanical parametric amplification and thermomechanical noise squeezing. Phys Rev Lett. 1991;67:699.

86. Szorkovszky A, Brawley GA, Doherty AC et al. Strong thermomechanical squeezing via weak measurement. Phys Rev Lett. 2013;110:184301.

87. Gardiner CW, Collett MJ. Input and output in damped quantum systems: quantum stochastic differential equations and the master equation. Phys Rev A. 1985;31:3761-74.

Submit your manuscript to a SpringerOpen ${ }^{\circ}$ journal and benefit from:

- Convenient online submission

- Rigorous peer review

- Open access: articles freely available online

- High visibility within the field

- Retaining the copyright to your article

Submit your next manuscript at $\boldsymbol{\nabla}$ springeropen.com 